\title{
Evaluation of a Decellularization Protocol for the Development of a Decellularized Tracheal Scaffold
}

\author{
ZETTA DIMOU $^{1,2}$, EFSTATHIOS MICHALOPOULOS ${ }^{1}$, MICHALIS KATSIMPOULAS ${ }^{3}$, DIMITRIOS DIMITROULIS ${ }^{2}$, \\ GREGORY KOURAKLIS ${ }^{2}$, CATHERINE STAVROPOULOS-GIOKAS ${ }^{1}$ and PERIKLIS TOMOS ${ }^{4}$ \\ ${ }^{1}$ Hellenic Cord Blood Bank, Biomedical Research Foundation of Academy of Athens, Athens, Greece; \\ ${ }^{2}$ Second Department of Propaedeutic Surgery, University of Athens, \\ Medical School, Laiko General Hospital, Athens, Greece; \\ ${ }^{3}$ Experimental Surgery Center and Translational Research, \\ Biomedical Research Foundation of Academy of Athens, Athens, Greece; \\ ${ }^{4}$ Department of Thoracic Surgery, Attikon University Hospital, Athens, Greece
}

\begin{abstract}
Background/Aim: Currently, there are no effective solutions for the treatment of advanced disorders of the airways. The aim of this study was to assess the efficacy of a decellularization protocol of the trachea in order to produce a functional scaffold for serious clinical respiratory disorders. Materials and Methods: Rat tracheas were decellularized using a protocol which included constituents with chemical action. Histological analysis was performed in order to evaluate the efficacy of decellularization. Genetic material was assayed and the toxicity of the decellularization protocol was assessed. Results: Histological analysis confirmed the removal of the nuclear and cellular components of the decellularized tissue, as well as maintenance of the extracellular matrix. DNA quantification showed removal of the genetic material. Furthermore, the decellularization protocol did not induce any cytotoxicity on tracheal tissue. Conclusion: The decellularization protocol was effective for tracheal decellularization. The final aim, in the future, would be to create a tissue-engineered airway which will be able to function normally.
\end{abstract}

Respiratory diseases are the third leading cause of death, and specific diseases of the trachea are the fifth most common cause of death, according to the World Health Organization (1).

The trachea is a tube with composite structure consisting of a longitudinal muscle, $\mathrm{C}$-shaped cartilage rings and specific

This article is freely accessible online.

Correspondence to: Zetta Dimou, Ph.D. student, Hellenic Cord Blood Bank, Biomedical Research Foundation Academy of Athens, 4 Soranou Ephessiou Street, Athens 11527, Greece. Tel: +30 2106597340, Fax: +30 2106597342, e-mail: dimou.zeta@gmail.com

Key Words: Trachea, scaffold, tissue-engineering, decellularization. respiratory mucosa. It connects the larynx to the bronchi, serving as a conduit for respiration, while removing various tracheal and bronchial secretions $(2,3)$. Due to exposure to internal and external mechanical forces, the trachea is characterized by a remarkable flexibility and other special features that allow neck movement and lateral rigidity to withstand positive and negative pressure during breathing and other processes (4). Congenital malformations, trauma, cancer, inflammation, injury after prolonged intubation and autoimmune disorders are some of the causes leading to the need for reconstruction or tracheal transplantation. Acquired or iatrogenic injury to the airways is relatively frequent and is considered lethal for the patient (2).

To date, there are no universally effective solutions for the treatment of advanced airway disorders, especially in children. Some treatment options include tracheoplasty, tracheal resection, and use of a stent or T-tube. Although tracheoplasty is the primary therapy for children, in some adults it can lead to recurrent stenosis (5). Tracheal resection is the only treatment for patients with various malignancies or benign disease. However, the length which can be resected is limited to $30 \%$ of the tracheaI length in children and $50 \%$ in adults. Lesions larger than these can be dealt with using stents or T-tubes, although drawbacks such as infection or displacement have been reported (2). When the above therapeutic approaches cannot be applied then only innovative solutions, such as tissue engineering techniques, become necessary in order to create functional tissue.

Tissue engineering has demonstrated its feasibility in experimental and clinical settings. Tissue engineering applies the principles and methods of biotechnology, materials science, transplantation of cells and life sciences, in an effort to develop in vitro biological substitutes able to restore, maintain or improve tissue and organ function $(6,7)$. Decellularization is one of the main methods of this domain. 
A decellularization protocol can be based on chemical, mechanical or enzymatic action of its components. The final goal of this process is the removal of nuclear materials and the preservation of the extracellular matrix of the tissue.

The aim of this study was the evaluation of a current decellularization protocol on rat trachea $(8,9)$. To our knowledge, this decellularization protocol has never been used in tracheal scaffold production. The ultimate goal of this study was to decellularize the rat trachea effectively, preserving the ultrastructure and minimizing decellularization cycles so as to develop a tracheal scaffold which could be used as a substitute in respiratory diseases. Decellularized trachea might serve as an alternative form for developing human implants.

\section{Materials and Methods}

Animal model. In our study, D/A male rats ( $\mathrm{n}=30$ ) weighing 220-300 g (Experimental Surgery Center and Translational Research, Biomedical Research Foundation of Academy of Athens, Athens, Greece). were used as donors of tracheal allografts. The current protocol for using animals was authorized by the Competent Regional Veterinary Authority (Ref. Number 1152/12-5-2015), in accordance with European Directive 2010/63 and National Legislation on the protection of animals used for scientific purposes (Presidential Decree 56/2013).

Tracheal harvesting and decellularization procedure. The entire length of rat tracheas $(n=18)$ was harvested and initially treated in $1 \times$ phosphate-buffered saline (PBS). The decellularization protocol was based on the chemical action of detergents and included solutions of 3[(3-cholamidopropyl) dimethylammonio]-1-propanesulfonate (CHAPS), sodium dodecyl sulfate (SDS) and $\alpha$-minimum essential medium supplementation ( $\alpha$-MEM)/fetal bovine serum (FBS) $(8,9)$. For each decellularization cycle, the isolated trachea samples were placed in CHAPS solution ( $8 \mathrm{mM}$ CHAPS, $1 \mathrm{M} \mathrm{NaCl}$, and $25 \mathrm{mM}$ EDTA diluted at $1 \mathrm{x}$ PBS; $\mathrm{pH} 8$ ) for $22 \mathrm{~h}$ at room temperature followed by washing in PBS $1 \times$ for 30 min at $4^{\circ} \mathrm{C}$. Tracheas were then placed in SDS solution (1.8 mM SDS, $1 \mathrm{M} \mathrm{NaCl}$, and $25 \mathrm{mM}$ EDTA diluted at $1 \times \mathrm{PBS} ; \mathrm{pH} 8$ ) for $22 \mathrm{~h}$ at room temperature followed by washing with PBS $1 \times$ for 30 min at $4^{\circ} \mathrm{C}$. Tracheas were finally placed in $\alpha-\mathrm{MEM} / 40 \% \mathrm{FBS}$ at $37^{\circ} \mathrm{C}$ for $48 \mathrm{~h}$. The above steps were carried out under constant shaking and were repeated three times.

Histological analysis. Histological analysis was performed to evaluate the efficacy of the decellularization protocol. For this purpose, native $(n=6)$ and decellularized $(n=6)$ tracheas were fixed in $10 \%$ neutral formalin, paraffin embedded and sectioned into 5 $\mu \mathrm{m}$ sections. The slides were then deparaffinized and stained with hematoxylin and eosin (H\&E; Sigma-Aldrich,Inc., St Louis, MO,USA) for nuclear and cellular material. Sections were also stained with 4',6-diamidino-2-phenylindole (DAPI; Invitrogen, Thermo Fisher Scientific, CA, USA) for nuclear material. LEICA DM RA2 (Leica, Wetzlar, Germany) fluorescence microscopy was used in order to evaluate the samples. Tracheal samples were also stained with toluidine blue (Sigma-Aldrich) for sulphated glycosaminoglycan content and Masson's Trichrome (SigmaAldrich) for collagen, nuclear and muscle content. Finally, elastic
Van Gieson (Sigma-Aldrich) staining was performed for the evaluation of elastin content. Histological samples were evaluated and images were obtained using a LEICA DM LS2(Leica, Wetzlar, Germany) optical microscope. Due to the fact that the tracheaI thickness varies along its length, different parts of the trachea were histologically evaluated as mentioned above, in order to carry out an integrated and reliable analysis.

DNA quantification. DNA quantification was performed in native $(n=6)$ and decellularized $(n=6)$ tracheas. According to the findings of previous studies, a recommended amount of $<50 \mathrm{ng}$ dsDNA $/ \mathrm{mg}$ matrix was considered to be a reliable criterion confirming the effectiveness of the decellularization protocol (10).

Native and decellularized tracheas were weighed and were digested using Proteinase K supplemented with PBS (Sigma Aldrich) and lysis buffer $(0.1 \mathrm{M}$ Tris $\mathrm{pH} 8,0.2 \mathrm{M} \mathrm{NaCl}, 5 \mathrm{mM}$ EDTA, $0.4 \%$ SDS) overnight at $55^{\circ} \mathrm{C}$, followed by DNA isolation. DNA was isolated from the entire trachea using a protocol which is based on solutions of phenol/chloroform/isoamyl alcohol. DNA quantification per milligram dry tissue was performed using Quantifluor dsDNA System E2670 Promega (Glomax; Promega, Madison, WI, USA) according to the manufacturer's instructions.

Cytotoxicity test. Two assays were performed in order to determine the cytotoxicity of the decellularization protocol. The first was a contact test of cells (11) with decellularized trachea and the second was an extract cytotoxicity assay regarding the ATP metabolism of cells. Wharton's jelly mesenchymal stromal cells (WJ MSCs) from human umbilical cord were used in order to perform the above tests due to their efficacy in differentiating into different cell populations such as epithelial or endothelial cells. Fresh umbilical cords were obtained and used after written informed consent from the mothers and were processed in less than 48 hours (12).

For the contact test, decellularized tracheas $(n=2)$ were cut into segments and placed on 6-well plates with WJ MSCs. Different culture procedures were performed with WJ MSCs: i) basic culture medium ( $\alpha$-MEM, $15 \%$ FBS, penicillin, streptomycin) as a negative control sample; ii) basic culture medium with decellularized trachea; and iii) basic culture medium with $10 \%$ dimethylsulfoxide (DMSO) in order to cause cytotoxicity, as a positive control sample. The samples were incubated at $37^{\circ} \mathrm{C}$ with $5 \% \mathrm{CO}_{2}$ for 24 hours and cell growth was evaluated by observing morphology and confluency using a LEICA DM LS2 (Leica, Germany) optical microscope.

ATP Colorimetric/Fluorometric Assay Kit (Sigma-Aldrich) was used for the evaluation of metabolic activity of cells which were cultured with digested decellularized trachea. Tracheas $(n=4)$ were digested using $1 \mathrm{ml}$ of $\alpha$-MEM supplemented with Proteinase $\mathrm{K}$ overnight. Proteinase $\mathrm{K}$ inactivation was achieved by incubation at $95^{\circ} \mathrm{C}$ for $5 \mathrm{~min}$. Decellularized tracheas were then placed in a 6-well plate with WJ MSCs and incubated until confluent. Cultures containing only WJ MSCs with $\alpha$-MEM were used as negative control samples. ATP concentration was determined by phosphorylating glycerol, resulting in a colorimetric $(570 \mathrm{~nm})$ product proportional to the amount of ATP present.

Statistics. Statistical analysis was performed using GraphPad Prism v 6.01 (GraphPad Software, San Diego, CA, USA). DNA and ATP content were analyzed using Mann-Whitney test. Statistically significant difference was considered when the $p$-value was less than 0.05 . Data are reported as the mean \pm standard deviation. 

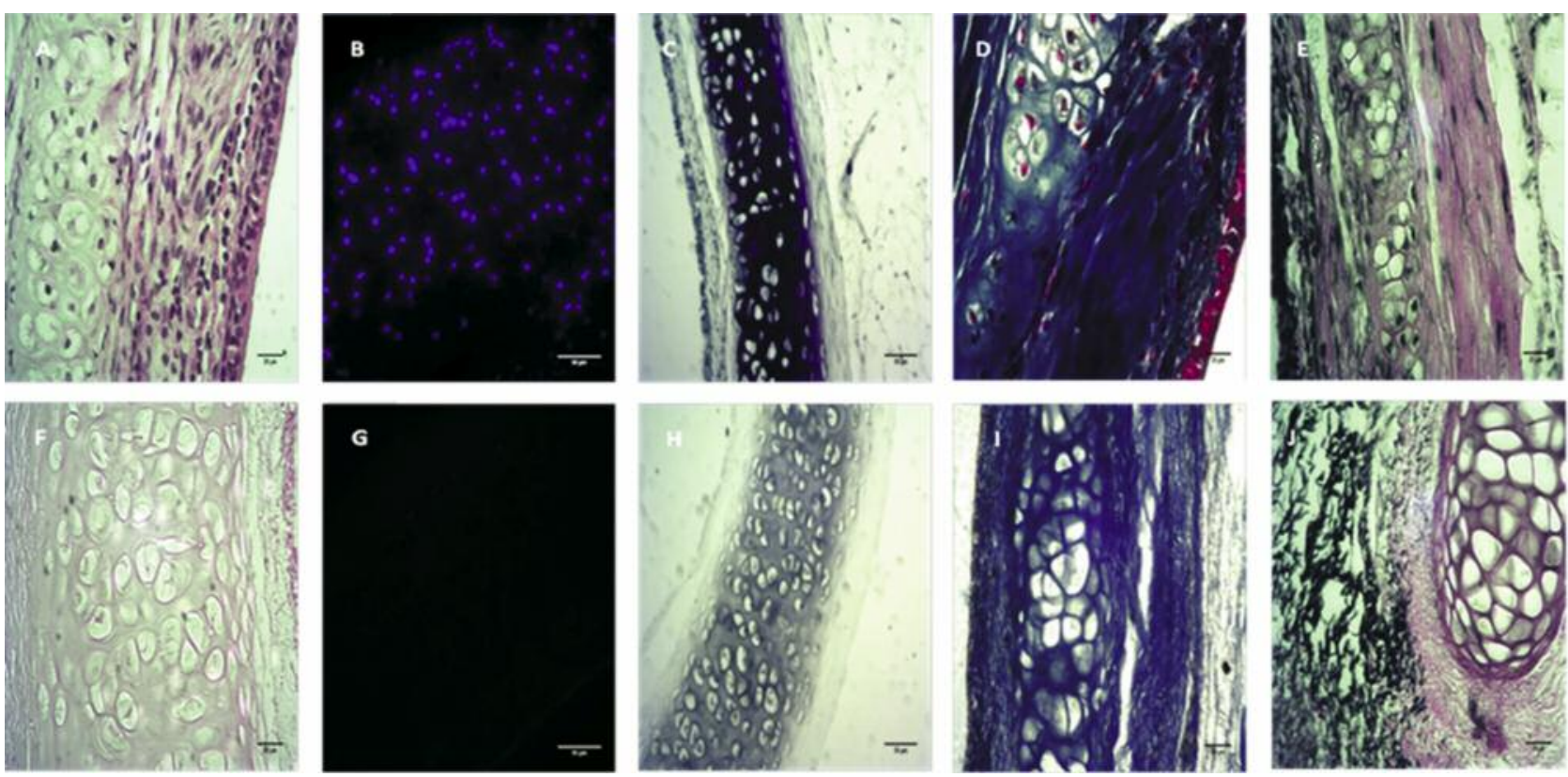

Figure 1. Histology of native (upper row) and decellularized (lower row) tracheal samples stained with hematoxylin and eosin $(A, F)$, $4^{\prime}, 6$-diamidino2-phenylindole $(B, G)$, toluidine blue $(C, H)$, Masson's trichrome $(D, I)$ and elastic Van Gieson $(E, J)$ after three cycles of decellularization at $20 \times$ $(C, H)$ and $40 \times(A, B, D, E, F, G, I, J)$ magnification.

\section{Results}

Efficacy of decellularization protocol. H\&E stain showed complete removal of nuclear and cellular materials after three cycles of decellularization compared to native samples. It was observed that the extracellular matrix remained intact (Figure 1A and F).

DAPI results showed complete nuclear removal from decellularized tracheas after three cycles of decellularization compared to native tissues (Figure 1B and G). In addition, it was shown that sulphated glycosaminoglycans, collagen and elastin were preserved in decellularized tissues (Figure 1C$\mathrm{E}$, and $\mathrm{H}-\mathrm{J}$ ), which were stained with toluidine blue (SigmaAldrich), Masson's Trichrome (Sigma-Aldrich) and elastic Van Gieson (Sigma-Aldrich) respectively.

DNA quantification. DNA quantification results after three cycles of decellularization showed that DNA content in decellularized tracheas was $4.1 \mathrm{ng}$ DNA/mg dry tissue, while in native tissue it was $722.9 \mathrm{ng}$ DNA/mg dry tissue (Figure 2). It appears that only $0.56 \%$ of the initial DNA remained in the decellularized samples. DNA content in decellularized tissues was significantly reduced compared to native tissue $(p=0.002)$.

Cytotoxicity assay. WJ MSCs cultured in basic culture medium with decellularized trachea grew and expanded at a

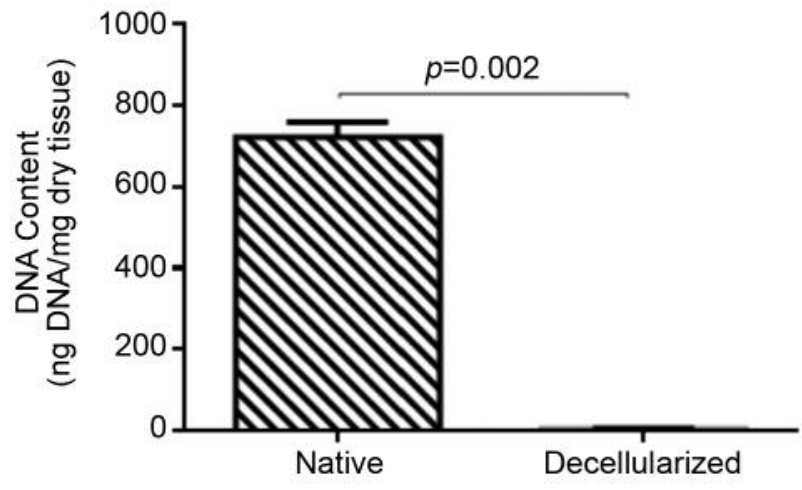

Figure 2. DNA quantification of native and decellularized tracheal samples.

similar rate compared to cells that were cultured only in basic culture medium (Figure 3A and B). However, apoptosis was observed in samples that were cultured in basic culture medium with 10\% DMSO (Figure 3C).

Furthermore, cells which were cultured with $\alpha$-MEM containing decellularized trachea appeared to metabolize ATP to an even greater rate than cells that were cultured only with $\alpha$-MEM (Figure 4), although there was no statistically significant difference between the two $(p=0.114)$. 

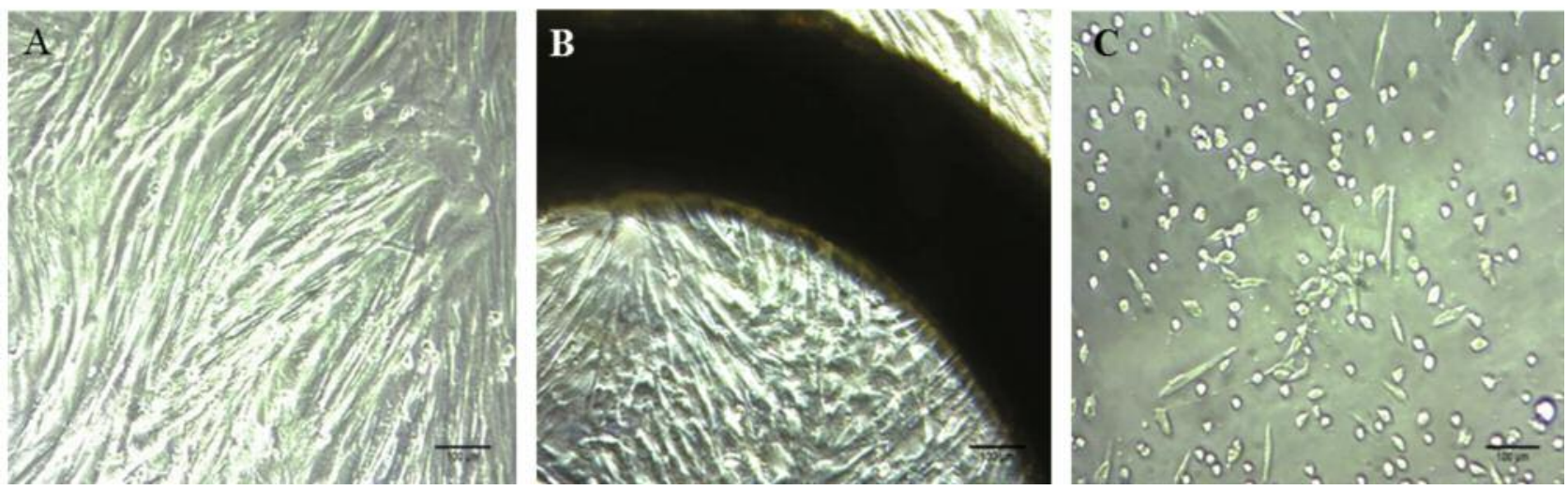

Figure 3. Contact cytotoxicity assay. Growth of Wharton's jelly mesenchymal stromal cells cultured in basic culture medium (A), basic culture medium with decellularized trachea $(B)$, basic culture medium and 10\% dimethylsulfoxide (C). Magnification 10x.

\section{Discussion}

The aim of this study was to evaluate a previously described decellularization protocol, which was first used on tracheal tissue $(8,13)$. The ultimate goal of this study was to decellularize rat trachea effectively, preserving the ultrastructure and minimizing the number of decellularization cycles required. An effective decellularized tissue should be characterized by complete removal of nuclear and cellular materials and preservation of extracellular matrix (14).

Conventional tracheal transplantation has some obvious drawbacks. The donor and the recipient should have matching HLA profiles but even in this case, these patients needs life-long immunosuppressive medication. An ideal scaffold for transplantation should fulfill specific requirements such as biocompatibility, non-immunogenicity and bioactivity. Scaffolds should be a model for tissue development in three dimensions, maintain and promote the growth of relevant cells or tissue, exhibit proper microarchitecture and functional properties, possess appropriate biomedical strength for the intended purpose, as well as functional vasculature to provide oxygen and nutrients. In addition, scaffolds must be completely safe for the body, non-toxic, non-tumorigenic and sterile (15).

In this study, rat trachea was decellularized according to the protocol which was based on the chemical action of its detergents. Only three cycles of the decellularization protocol were applied in order for the components to be removed and the trachea matrix to be preserved. After three cycles of decellularization, histological analysis and immunofluorescence showed complete absence of cellular and nuclear components while the extracellular matrix remained intact (Figure 1). Furthermore, concerning quantification of DNA, the results confirmed the efficacy of

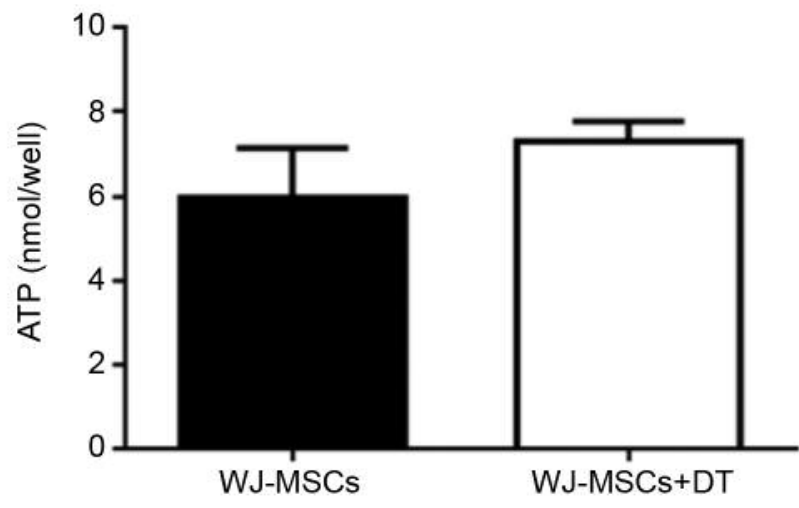

Figure 4. ATP metabolism of Wharton's jelly mesenchymal stromal cells (WJ MSCs) cultured in $\alpha-M E M$ alone and $\alpha-M E M$ with decellularized trachea tissue (DT). No significant difference in ATP metabolism was observed between native and decellularized trachea $(p=0.114)$.

the decellularization protocol. In particular, DNA quantification showed that only $0.6 \%$ of the initial amount of DNA from native trachea remained in the decellularized tissue. According to the findings of a previous study, the presence of $<50 \mathrm{ng}$ dsDNA/mg matrix is considered to be a reliable criterion for confirming the effectiveness of the decellularization protocol (10).

Most decellularization protocols which have been used previously are based on enzymatic and chemical action of their components. Zang et al. decellularized rat trachea using a modified detergent-enzymatic treatment. Rat trachea treated with five detergent-enzymatic treatment cycles demonstrated good preservation of the extracellular matrix structure (16). Another study presented the characterization of rat trachea decellularized under dynamic conditions, implementing a 
detergent-enzymatic method. Specifically, six cycles were performed to generate a tracheal scaffold which was histologically and structurally similar to the native one (17). The main difference from this study was the duration of decellularization process. Decellularization time and treatment cycles are important factors, which determine the preservation of trachea, because it is recognized that all methods of decellularization result in disruption of the architecture and potential loss of surface structure and composition (14).

Furthermore, cytotoxicity effect of decellularization agents was evaluated here. WJ MSCs were selected for cytotoxicity assays due to their potential to differentiate into cell populations such as epithelial or endothelial cells (18). Cytotoxicity test showed that decellularized trachea tissues did not induce cytotoxicity in WJ MSCs. The aim of the contact test (11) was to determine the success of adhesion to decellularized trachea and plastic surface which would not be feasible if there was any cytotoxic activity in the culture. Cells cultured with decellularized trachea grew and expanded at a similar rate to cells cultured with native trachea. Furthermore, cells which were cultured in $\alpha$-MEM with decellularized trachea appeared to present greater proliferative and metabolic capacity compared to the control group. Our study gave results similar to studies conducted on trachea decellularization using other decellularization protocols $(10,16,17)$, widening perspectives in regeneration of tissue-engineered trachea.

The decellularization protocol appeared to be effective in the decellularization of trachea tissues, however, further study is required in laboratory testing such as biomechanical evaluation, and quantification of glycosaminoglycans and collagen. The final aim, in the future, would be to create a cell-based, tissue-engineered airway, without using a bioreactor, which will be able to operate normally without the risk of an immune response. Supporting evidence by Kajbafzadeh and colleagues (10) indicated that it was possible to implant tracheas in nude mice using the body as a natural bioreactor for tissue recellularization. Based on the above research, future studies should be oriented to transplantation of decellularized trachea in animal models, evaluation of tissue recellularization and monitoring of the immune response.

\section{Conclusion}

In conclusion, creating a tissue-engineered trachea for the treatment of severe diseases of the respiratory tract without using a bioreactor is a challenge. In this study it was found that the decellularization protocol, which was used for the first time for the decellularization of rat trachea, was successful. Long-term studies are necessary for proper evaluation of the in vivo efficacy and reliability of decellularized scaffolds for use in the future for the treatment of patients.

\section{Acknowledgements}

The Authors are grateful to Mrs Vlahou Antonia and Mr. Zoidakis Jerome, from the Biotechnology Division of the Biomedical Research Foundation Academy of Athens, for their support of our study.

\section{Conflicts of Interest}

The Authors declare no conflict of interest exists in regard to this study.

\section{References}

1 World Health Organization, WHO Methods and Data Sources for Country-level Causes of Death 2000-2012, Global Health Estimates Technical Paper WHO/HIS/HSI/GHE/2014.7, Department of Health Statistics and Information Systems, World Health Organization, Geneva, 2014. Available at: http:// www.who.int/healthinfo/statistics/GlobalCOD_method.pdf?ua= 1, last accessed 03/12/2018.

2 Crowley $\mathrm{C}$, Birchall $\mathrm{M}$ and Seifalian AM: Trachea transplantation: From laboratory to patient. J Tissue Eng Regen Med 9(4): 357-367, 2014.

3 Roomans GM: Tissue engineering and the use of stem/progenitor cells for airway epithelium repair. Eur Cell Mater 19: 284-299, 2010.

4 Grillo HC: Tracheal replacement: A critical review. Ann Thorac Surg 73: 1995-2004, 2002.

5 Elliott MJ, De Coppi P, Speggiorin S, Roebuck D, Butler CR, Samuel E, Crowley C, McLaren C, Fierens A, Vondrys D, Cochrane L, Jephson C, Janes S, Beaumont NJ, Cogan T, Bader A, Seifalian AM, Hsuan JJ, Lowdell MW and Birchall MA: Stemcell-based, tissue engineered tracheal replacement in a child: A 2year follow-up study. Lancet 380(9846): 994-1000, 2012.

6 Langer $\mathrm{R}$ and Vacanti JP: Tissue engineering. Science 260(5110): 920-926, 1993.

7 Vacanti JP and Langer R: Tissue engineering: the design and fabrication of living replacement devices for surgical reconstruction and transplantation. Lancet 354(suppl 1): S132S134, 1999.

8 Gui L, Muto A, Chan SA, Breuer CK and Niklason LE: Development of decellularized human umbilical arteries as small-diameter vascular grafts. Tissue Eng 15(9): 2665-2676, 2009.

9 Petersen TH, Calle EA, Zhao L, Lee EJ, Gui L, Raredon MB, Gavrilov K, Yi T, Zhuang ZW, Breuer C, Herzog E and Niklason LE: Tissue engineered lungs for in vivo implantation. Science 329: 538-541, 2010.

10 Kajbafzadeh AM, Sabetkish S, Sabetkish N, Muhammadnejad S, Akbarzadeh A, Tavangar SM, Mohseni MJ and Amanpour S: In vivo trachea regeneration: Fabrication of a tissue-engineered trachea in nude mice using the body as a natural bioreactor. Surg Today 45(8): 1040-1048, 2015.

11 Mallis P, Michalopoulos E, Dimitriou C, Kostomitsopoulos N and Stavropoulos-Giokas C: Histological and biomechanical characterization of decellularized porcine pericardium as a potential scaffold for tissue engineering applications. BioMedical Mater Eng 28: 477-488, 2017. 
12 Chatzistamatiou TK, Papassavas AC, Michalopoulos E, Gamaloutsos C, Mallis P, Gontika I, Panagouli E,Koussoulakos SL and Stavropoulos-Giokas C: Optimizing isolation culture and freezing methods to preserve Wharton's jelly's mesenchymal stem cell (MSC) properties: An MSC banking protocol validation for the Hellenic Cord Blood Bank. Transfusion 54(12): 3108-3120, 2014.

13 Mallis P, Gontika I, Poulogianopoulos T, Zoidakis J, Vlachou A, Chatzistamatiou T, Michalopoulos E and Stavropoulos-Giokas C: Evaluation of decellularization in umbilical cord artery. Transplant Proc 46: 3232-3239, 2014.

14 Crapo PM, Gilbert TW and Badylak SF: An overview of tissue and whole organ decellularization processes. Biomaterials 32(12): 3233-3243, 2011.

15 Fishman JM, Lowdell M and Birchall MA: Stem cell-based replacements-Airway and lung tissue engineering. Semin Pediatr Surg 23: 119-126, 2014.
16 Zang $\mathrm{M}$, Zhang Q, Chang EI, Mathur $\mathrm{AB}$ and $\mathrm{Yu} \mathrm{P}$ : Decellularized tracheal matrix scaffold for tissue engineering. Plast Reconstr Surg 130(3): 532-540, 2012.

17 Baiguera S, Del Gaudio C, Kuevda E, Gonfiotti A, Bianco A and Macchiarini P: Dynamic decellularization and cross-linking of rat tracheal matrix. Biomaterials 35(24): 6344-6350, 2014.

18 Spees JL, Olson SD, Ylostalo J, Lynch PJ, Smith J, Perry A, Peister A, Wang MY and Prockop DJ: Differentiation, cell fusion, and nuclear fusion during ex vivo repair of epithelium by human adult stem cells from bone marrow stroma. Proc Natl Acad Sci USA 100(5): 2397-2402, 2003.

Received October 31, 2018

Revised November 30, 2018

Accepted December 4, 2018 